\title{
Clinical experience in idiopathic pulmonary fibrosis: a retrospective study
}

\section{Julien Guiot, Bernard Duysinx, Laurence Seidel, Monique Henket, Fanny Gester, Olivier Bonhomme, Jean-Louis Corhay \& Renaud Louis}

To cite this article: Julien Guiot, Bernard Duysinx, Laurence Seidel, Monique Henket, Fanny Gester, Olivier Bonhomme, Jean-Louis Corhay \& Renaud Louis (2017): Clinical experience in idiopathic pulmonary fibrosis: a retrospective study, Acta Clinica Belgica, DOI: 10.1080/17843286.2017.1399228

To link to this article: https://doi.org/10.1080/17843286.2017.1399228

册 Published online: 10 Nov 2017.

Submit your article to this journal $\pi$

Џ Article views: 31

Q View related articles $\longleftarrow$

View Crossmark data $[7$ 


\title{
Clinical experience in idiopathic pulmonary fibrosis: a retrospective study
}

\author{
Julien Guiot ${ }^{a}$, Bernard Duysinx $x^{\mathrm{a}}$, Laurence Seidel ${ }^{\mathrm{b}}$, Monique Henket ${ }^{\mathrm{a}}$, Fanny Gester ${ }^{\mathrm{a}}$, Olivier Bonhomme ${ }^{\mathrm{a}}$, \\ Jean-Louis Corhay and Renaud Louis ${ }^{a}$
}

aPneumology Department, CHU Liège, Domaine universitaire du Sart-Tilman, Liège, Belgium; ${ }^{b}$ Department of Medico-Economy and Biostatistics, Domaine universitaire du Sart-Tilman, Liège, Belgium

\begin{abstract}
Introduction: Idiopathic pulmonary fibrosis (IPF) is a rare lung disease with an increased incidence since the last few years. Here, we report our eight-year clinical experience in CHU of Liège, Belgium.

Methods: We have studied retrospectively patients recruited from our ambulatory care polyclinic at CHU of Liège from 1 January 2009 to 1 January 2017. We have excluded all patients treated with a specific anti-fibrotic therapy due to incomplete follow-up. The diagnosis of IPF was made according to the ATS/ERS international recommendations (2015).

Results: Out of the 114 patients initially selected, 82 cases were found to be suitable for the analysis. The average age was $71.1 \pm 9.35$ years with a male predominance. The median survival was 43.7 months (23.6-71.7) with a majority (45\%) of patients in the group II of the GAP index. The median rate of annual decline in diffusion capacity of CO (DLCO) was $11 \%$, whereas the sub analysis for group III (according to GAP index) showed a decrease annual rate of $30 \%$.

Conclusion: Our results are in keeping with the literature. One of our major finding is that patients in GAP III exhibit an annual rate of mortality of $42 \%$ and a median annual decline in DLCO of $30 \%$. This observation highlights the fact that this specific subgroup of patients presents a high risk of morbi-mortality.
\end{abstract}

\section{KEYWORDS}

Idiopathic pulmonary

fibrosis; interstitial lung

diseases; prognosis

\section{Introduction}

Idiopathic pulmonary fibrosis (IPF) is a rare lung disease of unknown origin which leads rapidly to death $[1,2]$. Epidemiological studies suggest that the incidence of IPF has been increasing steadily over the last two to three decades [3]. Its incidence is evaluated to 2-29/100.000 people [4-6]. However, recent study from USA has shown that incidence rate may be as high as 76.4/100.000 in subjects above 75 years (based on a broad IPF definition) [7].The sex ratio is in favor of male with an increase prevalence of smokers (or ex-smokers with a smoking history of more than 20 pack years). There are also familial forms, which are associated with mutations thought to be autosomic dominant with an incomplete penetrance (mainly TERT/TERC and SFTPC) [8].

The disease is generating a progressive fibrosing process exclusively limited to the lungs, which is very specific particularity in comparison with other interstitial lung diseases. The symptomatic presentation is variable as well as the clinical course of the disease, which is ranging from a slow progressive evolution to a rapid decline or with an acute exacerbation pattern. Classically, the symptoms increase until pulmonary insufficiency, which is the main cause of death. Acute exacerbation in IPF is a rare complication secondary to one hyper inflammatory state associated with a highly increased mortality rate.

Although the etiology and the pathophysiology of IPF are still incompletely understood, two antifibrotic drugs, pirfenidone and nintedanib, have recently proven to be effective in slowing down disease progression, and, are now approved as treatments $[9,10]$. Clinical management of IPF remains difficult due to a lack of accurate indicators of disease progression, and an absence of simple short-term measures of therapeutic response [11-13]. Recently, new data studying the pooled analysis of the previous studies with pirfenidone identified a benefit on mortality in IPF patients [14].

The aim of our work was to analyze our eight-year clinical experience in IPF in the CHU of Liège (Belgium) and to confirm whether our clinical data are confident with the literature.

\section{Methods}

We retrospectively studied patients recruited from our ambulatory care policlinic at CHU from 1 January 2009 to 1 January 2017. We excluded all patients treated with specific anti-fibrotic therapy. The diagnosis of IPF was made according to the international recommendations 
of the ATS/ERS $[1,15]$ using the respiratory function tests, high-resolution computed tomography scan (probable UIP pattern), bronchoalveolar lavage (when available), as well as the clinical history of the patient. We excluded all other causes of interstitial lung disease (such as asbestosis, hypersensitivity pneumonitis, pneumonia associated with connective tissue disease or toxic pneumonitis). We combined the different results for the diagnosis. All cases were discussed in a multidisciplinary group about interstitial lung diseases composed of a pulmonologist, a specialist in pulmonary rehabilitation, a rheumatologist, a radiologist, a pathologist and a specialist in occupational medicine.

\section{Pulmonary function tests}

We performed pulmonary function tests (PFT) in our routine respiratory laboratory of $\mathrm{CHU}$ of Liège. All spirometric tests performed for this study were measured using the pneumotachograph JaegerMasterlab system (Erich Jaeger GmbH, Wuzburg, Germany). The forced expiratory volume in one second (FEV1) and forced vital capacity (FVC) were measured in accordance with the recommendations of the European Respiratory Society (ERS). The results were expressed in milliliter and percentage of predicted values. The Tiffeneau index or FEV1/FVC was expressed in percent. The total lung capacity (TLC) was measured by body plethysmography according to ERS recommendations (Erich Jaeger $\mathrm{GmbH}$, Wuzburg, Germany). The diffusion capacity of CO (DLCO) and the report DLCO/VA were measured by the single-breath carbon monoxide gas transfer method and expressed as percentage of predicted values (Sensor Medics $2400 \mathrm{He} / \mathrm{CO}$ Analyzer System, Bilthoven, the Netherlands).

\section{GAP score}

One easy, simple, and useful index that has been proposed for predicting IPF prognosis is the GAP index risk prediction model, which includes four factors: gender $(G)$, age $(A)$, and two lung physiologic $(P)$ variables (FVC (\% pred) and DLCO (\% pred)) (Table 1) [16]. Patients were stratified based on GAP score: GAP I are patients with $0-3$ points, GAP II are patients with 4 and 5 points,

Table 1. GAP index score for idiopathic pulmonary fibrosis.

\begin{tabular}{|c|c|c|c|c|c|c|}
\hline \multicolumn{3}{|c|}{ G (Gender) } & \multicolumn{4}{|c|}{$A$ (Age) } \\
\hline Female & & Male & $\leq 60$ & $61-65$ & & $\geq 65$ \\
\hline$\underline{0}$ & & 1 & 0 & 1 & & 2 \\
\hline \multicolumn{7}{|c|}{$P$ (Physiology) } \\
\hline \multicolumn{3}{|c|}{ FVC post-BD (\% predicted) } & \multicolumn{4}{|c|}{ DLCO (\% predicted) } \\
\hline$>75 \%$ & $50-75 \%$ & $<50 \%$ & $>55 \%$ & $36-55 \% \leq$ & $\leq 35 \%$ & $\begin{array}{l}\text { Cannot } \\
\text { perform }\end{array}$ \\
\hline
\end{tabular}

\begin{tabular}{llllllr}
0 & 1 & 2 & 0 & 1 & 2 & 3 \\
\hline Notes: adapted from Ley et al. [16]. GAP I: & $0-3$ points, GAP II: 4 and 5
\end{tabular} points, GAP III: 6-8 points. FVC: forced vital capacity; DLCO: diffusion lung capacity of $\mathrm{CO}$. and GAP III patients with 6-8 points. The patients without complete PFT available or without sufficient quality of collaboration for analysis were only listed in the all cohort and not included in the GAP sub-analysis.

\section{Statistical analysis}

Results are expressed as frequency tables for qualitative variables and as mean and standard deviation (SD) or as median and quartiles (Q1-Q3) for continuous variables. Comparisons between groups were done by chi-square test for qualitative variables and by Kruskal-Wallis test for continuous variables. Overall, survival was represented by a Kaplan-Meier curve. Survival between groups was compared by Cox regression model (HR and $95 \%$ confidence interval). To calculate the annual decrease of a parameter, a linear regression of this parameter on time since first EFR was applied for each patient. The annual decrease is calculated as the slope corrected by the number of days between the first and the last EFR and converted in percent per year. The annual decrease is assessed by the Wilcoxon sign-rank test. Comparison of annual decrease between groups was done by Kruskal-Wallis test. To compare groups two by two, a Bonferroni correction was applied. Results were significant at the $5 \%$ significance level $(p<0.05)$. All statistical analyses were carried out by SAS version 9.4 (SAS Institute, Cary, NC, USA) and figures by Rersion 3.2.2.

\section{Results}

We identify 114 untreated patients suffering from IPF and excluded 32 of them because of incomplete data-set.

\section{Subject demographic and functional characteristics}

Subjects' characteristics are listed in Table 2. The time of follow-up range from 1 to 92 months. The mean age of IPF patients was $71.1 \pm 9.35$ years with a male dominance $(69.5 \%)$. Spirometric values were lower than predicted values with a restrictive pattern and a reduced DLCO as expected. The GAP I group present higher values for TLC, FVC, FEV1, and DLCO than GAP II and III, whereas the GAP II group exhibit higher values for FVC, FEV1, and DLCO than GAP III. GAP III group had a significantly lower DLCO/VA than groups I and II $(p<0.0013)$.

\section{Decline of PFTs}

We also studied the decline of PFTs separating patients in three groups according to their GAP score (Table 3). We've identified that the median annual rate of decline (in absolute values) for CPT, FVC, FEV1, and DLCO was $1.90,2.64,3.75$, and $10.80 \%$, respectively. Interestingly, in the GAP III group sub analysis, we identified that there 
Table 2. Subjects characteristics.

\begin{tabular}{|c|c|c|c|c|c|}
\hline & Total $^{\mathrm{a}}$ & Gap I & Gap II & Gap III & \\
\hline & Median (Q1-Q3) & Median (Q1-Q3) & Median (Q1-Q3) & Median (Q1-Q3) & \\
\hline & $n=82$ & $n=26(34 \%)$ & $n=35(45 \%)$ & $n=16(21 \%)$ & $p$-value \\
\hline Gender (M/F), $n$ & $55 / 27$ & $14 / 12$ & $27 / 8$ & $13 / 3$ & 0.080 \\
\hline Smokers (never/ex/current), \% & $26.7 / 52 / 21.3$ & $20.8 / 50.0 / 29.2$ & $23.5 / 47.1 / 29.4$ & $30.8 / 61.5 / 7.7$ & 0.61 \\
\hline Age (years) mean $\pm S D$ & $71.1 \pm 9.35$ & $66.7 \pm 11.80^{\mathrm{a}}$ & $71.80 \pm 6.72$ & $76.7 \pm 6.20^{a}$ & 0.0019 \\
\hline Follow-up (months) & $24.6(10.9-43.9)$ & $27.8(18.9-62.3)$ & $33.4(10.9-46.7)$ & $13.3(3.15-25.2)$ & 0.044 \\
\hline Range & 0-92 & $0-92$ & $0.6-91.3$ & $0.99-74$ & \\
\hline Dead (no/yes), \% & $48.8 / 51.1$ & $76.9 / 23.1^{\mathrm{a}, \mathrm{b}}$ & $42.9 / 57.1^{\mathrm{a}}$ & $18.8 / 81.2^{b}$ & 0.0007 \\
\hline Median survival (months) & $43.7(23.6-71.7)$ & $>92(70.5$ to $>92)$ & $43.7(24.6-70.8)$ & $21.4(4.9-26.1)$ & $<0.0001$ \\
\hline TLC \%pred & $77.0(61.0-92.0)$ & $92.0(81.0-101)^{\mathrm{a}, \mathrm{b}}$ & $74.0(61.0-85.0)^{\mathrm{a}}$ & $59.5(49.5-71.5)^{b}$ & $<0.0001$ \\
\hline FVC \%pred & $78.0(63.0-94.0)$ & $94.5(88.0-106)^{a, b}$ & $76.0(63.0-90.0)^{a, c}$ & $62.5(43.5-68.5)^{b, c}$ & $<0.0001$ \\
\hline FEV1 \%pred & $77.0(64.0-92.0)$ & $89.5(79.0-101)^{\mathrm{a}, \mathrm{b}}$ & $76.0(60.0-88.0)^{\mathrm{a}, \mathrm{c}}$ & $61.0(52.0-73.0)^{\mathrm{b}, \mathrm{c}}$ & $<0.0001$ \\
\hline DLCO \%pred & $45.5(34.0-60.0)$ & $62.0(46.0-69.0)^{a, b}$ & $43.0(36.0-53.0)^{a, c}$ & $26.0(25.0-28.0)^{b, c}$ & $<0.0001$ \\
\hline DLCO/VA \% & $72.5(59.5-89.0)$ & $80.0(65.0-94.0)^{\mathrm{b}}$ & $71.0(59.0-90.0)^{c}$ & $56.0(45.0-63.0)^{\mathrm{b}, \mathrm{c}}$ & 0.0013 \\
\hline
\end{tabular}

Notes: TLC: total lung capacity; FVC: forced vital capacity; FEV1: forced expired volume in one second; DLCO: diffusion lung capacity for CO; DLCO/VA: DLCO/ alveolar ventilation.

Bonferroni correction for group comparison. The differences are noticed with the same letter.

aWe had only 77 patients with full PFTs.

Table 3. Annual decline of the main physiological pulmonary parameters in our cohort of IPF patients.

\begin{tabular}{|c|c|c|c|c|c|c|}
\hline & \multirow{2}{*}{$\frac{\text { TLC }}{1.90(-6.45 \text { to } 11.1)}$} & \multirow{2}{*}{$\frac{\text { FVC }}{2.64(-6.56 \text { to } 7.12)}$} & \multirow{2}{*}{$\frac{\text { FEV1 }}{3.75(-4.99 \text { to } 7.62)}$} & \multirow{2}{*}{$\frac{\text { DLCO }}{10.8(-2.64 \text { to } 16.4)}$} & \multirow{2}{*}{$\frac{\text { DLCO/VA }}{6.20(-3.22 \text { to } 14.6)}$} \\
\hline \multicolumn{2}{|c|}{$\begin{array}{l}\text { Annual Decline (\%) } \\
\text { All } \quad \text { Median (01-03) }\end{array}$} & & & & & \\
\hline \multicolumn{2}{|c|}{ GAP I $\quad$ Median (Q1-Q3) } & $4.92(-5.06$ to 12.4$)$ & $5.27(-3.88$ to 6.99$)$ & $3.74(0.64-6.83)$ & $12.7(3.13-15.7)^{\mathrm{a}}$ & $6.02(0.16-9.45)$ \\
\hline \multirow{2}{*}{$\begin{array}{l}\text { GAP II } \\
\text { GAP III }\end{array}$} & Median (Q1-Q3) & $0.58(-6.24$ to 8.35$)$ & $1.33(-6.30$ to 6.62$)$ & $5.32(-5.60$ to 8.25$)$ & $5.83(-17.5 \text { to } 15.6)^{b}$ & $5.69(-5.32$ to 14.6$)$ \\
\hline & Median (Q1-Q3) & $-12.5(-53.1$ to -1.26$)$ & $-6.43(-26.6$ to 16.2$)$ & $-10.8(-15.2$ to 10.4$)$ & $30.2(20.6-40.9)^{a, b}$ & $29.1(27.2-35.6)$ \\
\hline
\end{tabular}

Note: significant reduction in DLCO between GAP III vs GAP I $(p=0.0012)$ and GAP III vs GAP II $(p=0.01)$.

${ }^{a}$ significant reduction in DLCO between GAP III vs GAPI $(p=0.0012)$

bsignificant reduction in DLCO between GAP III vs GAP II $(p=0.01)$.

was no real change in the pulmonary volumes. Conversely to that observation, there was an annual rate of DLCO decline around $30 \%$ underlying the rapid degradation of the gas exchanges in patients with the more severe condition. On the other hand, the GAP I group present an annual decline of the FVC around $5 \%$ of the initial absolute value ranging from -4 to $7 \%$ with an annual DLCO reduction around 13\% (Figure 1). Globally there are no differences between the three groups except for GAP III which present a significantly increased decline in DLCO $(p=0.01)$ compared to GAP I and II.

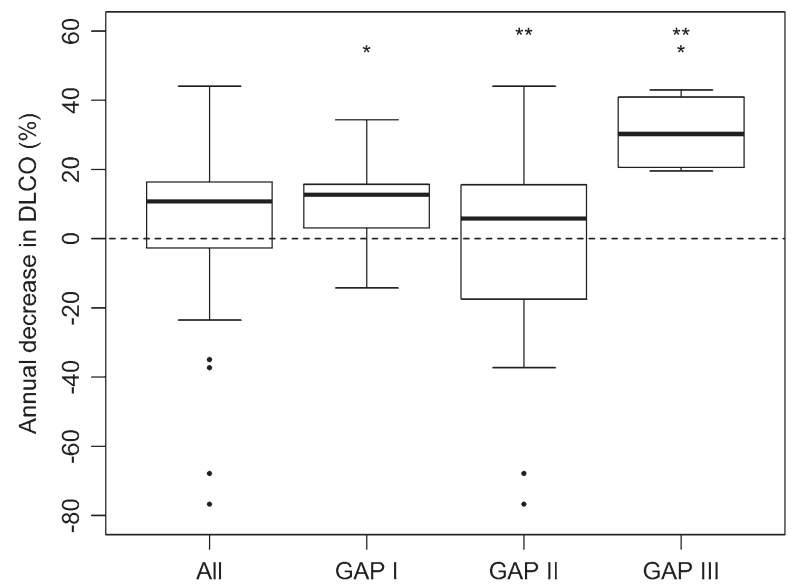

Figure 1. Annual decrease of DLCO (in absolute value) in the overall population of IPF and according to the GAP I, II, and III subgroups.

Notes: Bonferroni correction comparing GAP I vs. II vs. III showing a significant difference between group I vs. III $\left({ }^{*} p<0.017\right)$ and II vs. III $(* * 00.001)$.

\section{Survival according to the GAP index}

Concerning the survival data, it is widely known that median survival rate in IPF without specific therapy is around 3-5 years. In our experience, the median overall survival is 43.7 months (23.6-71.7). In our cohort of patients, based on the GAP index, we identified $34 \%$ of patients in group I; $45 \%$ in group II and $21 \%$ in group III.

The overall mortality is represented in Figure 2, while the Figure 3 is representing the mortality according to the GAP index classification. Based on the GAP index, we found a one-year mortality of 4,14 , and $42 \%$ in stages I, II, and III, respectively, whereas the three-year mortality was 17,39 , and $86 \%$, respectively (Table 4). Cox model identified a highly significant difference in mortality by separating patients with GAP score $(p=0.0002)$, where the GAP III group exhibit a very high mortality rate with an HR of 7.70 (2.88-20.6; $p<0.0001)$ and $2.68(1.31-5.46 ; p=0.0067)$ in comparison with GAP I and III, respectively. Patients in GAP I exhibit a lower mortality than GAP II and III $(p=0.0007)$.

\section{Hospitalizations}

The hospitalization rate is presented in Table 5 . The median hospitalization rate is four during the follow-up, whereas the median number of admission is bellow one for intensive care unit (ICU) and of two for emergency room (ER) admissions. 


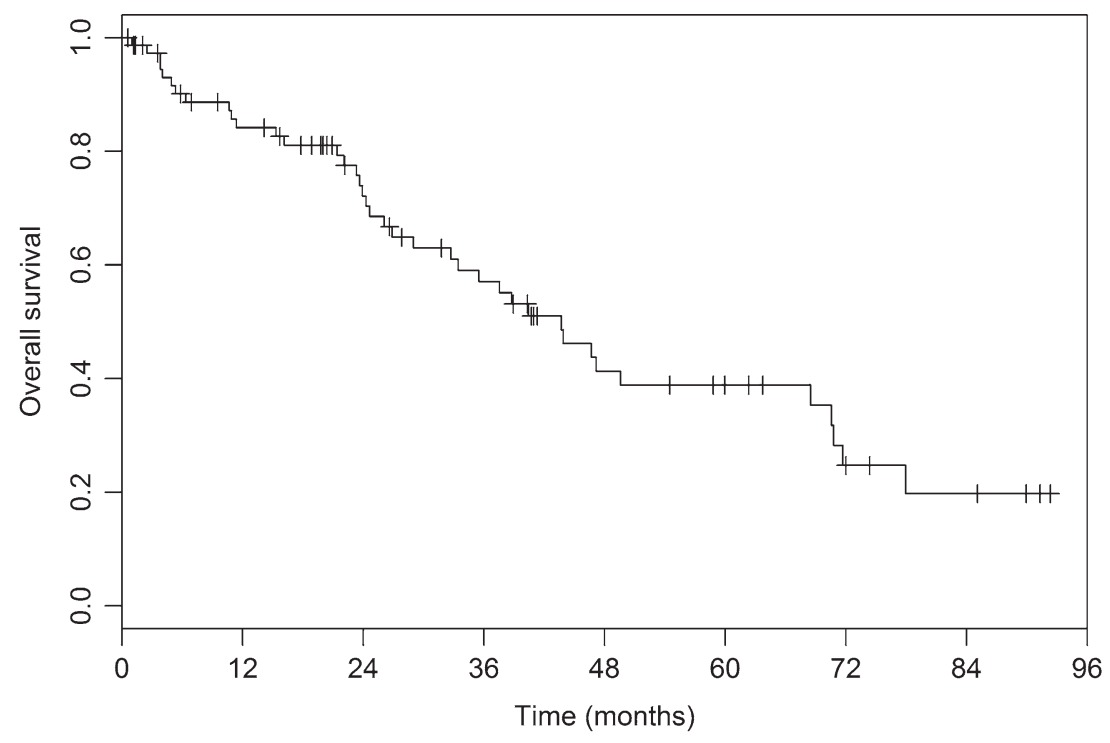

Figure 2. Overall survival of the cohort of IPF. Note: Kaplan-Meier curve.

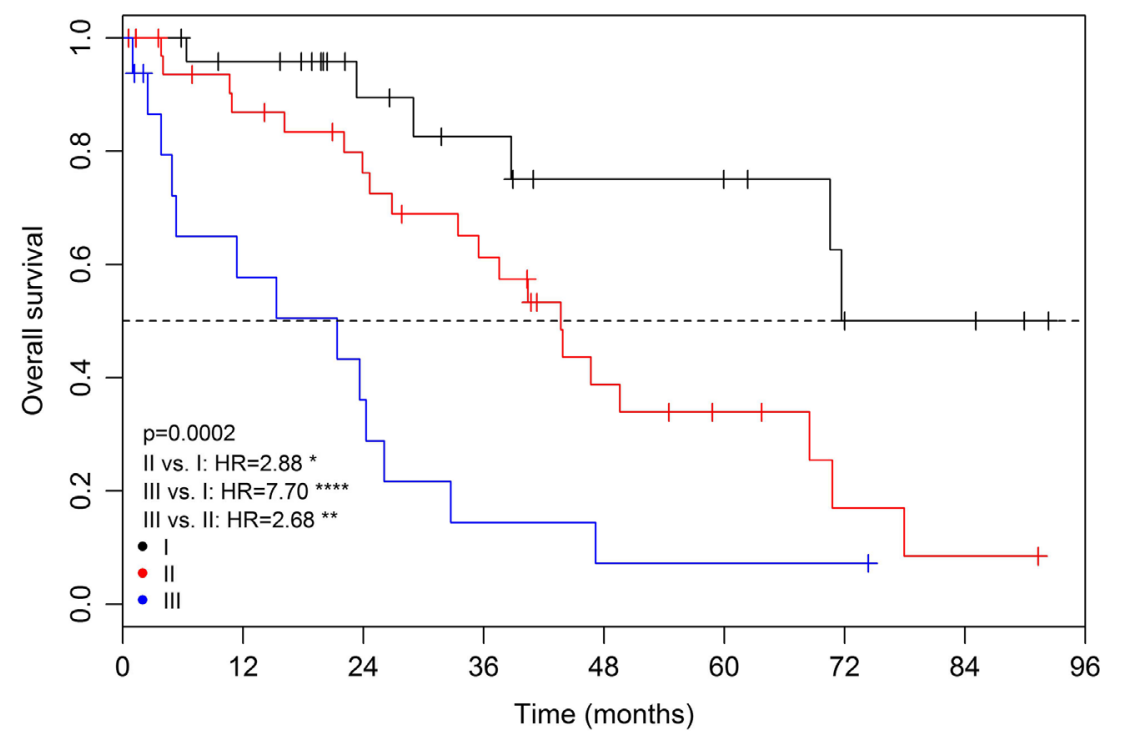

Figure 3. Survival of IPF cohort according to the GAP index.

Notes: Survival after a specific repartition in three groups according to GAP index. We identified a one-year mortality of 4, 14, and $42 \%$ in stages I, II, and III, respectively, whereas the three-year mortality was 17,39 , and $86 \%$, respectively.

Table 4. Mortality at 1 and 3 years in our cohort according to the GAP index group vs. what is given in the literature [16].

\begin{tabular}{lccc}
\hline & $\%$ of patients & $\begin{array}{c}\text { 1-y mortality (vs. } \\
\text { literature) }\end{array}$ & $\begin{array}{c}\text { 3-y mortality (vs. } \\
\text { literature) }\end{array}$ \\
\hline GAP I & $34 \%$ & $4 \%$ (vs. 6\%) & $17 \%$ (vs. 16\%) \\
GAP II & $45 \%$ & $14 \%$ (vs. 16\%) & $39 \%$ (vs. 42\%) \\
GAP III & $21 \%$ & $42 \%$ (vs. 39\%) & $86 \%$ (vs. 77\%) \\
\hline
\end{tabular}

\section{Discussion}

Here, we report our eight-year clinical experience of IPF in the university hospital of Liege showing that our data are in keeping with the literature.

The epidemiological characteristics are similar than those seen in the literature [1-7], which are classically elderly males with a history of smoking. However, we often encounter approximately $30 \%$ never-smoking IPF patients [17]. In our study, we confirm the male predominance with a mean age of 71 years, and $27 \%$ of the all population had never smoked. As expected, following the definition of the GAP index, we identify a altered FVC and DLCO increasingly from GAP I to GAP III. Interestingly, there was no difference between GAP II and III patients for TLC.

Concerning the survival data, it is widely known that median survival rate in IPF without a specific therapy is around 3-5 years. In our experience, the median overall survival is 43.7 months (23.6-71.7). We also have separated the patients according to the GAP index [16]. Our data are again consistent with the literature exhibiting a one-year mortality of 4,14 , and $42 \%$ in stages I, II, and 
Table 5. Hospitalizations in the IPF cohort.

\begin{tabular}{|c|c|c|c|c|c|c|c|c|c|}
\hline Variable & $N$ & Mean & SD & SE & Min & Q1 & Median & Q3 & Max \\
\hline Hospitalization & 103 & 5.17 & 5.85 & 0.58 & 0.0 & 2.0 & 4.00 & 7.0 & 41.0 \\
\hline ICU admission & 89 & 0.67 & 1.30 & 0.14 & 0.0 & 0.0 & 0.00 & 1.0 & 9.0 \\
\hline ER admission & 89 & 2.25 & 2.63 & 0.28 & 0.0 & 1.0 & 2.00 & 3.0 & 16.0 \\
\hline Total stay (days) & 89 & 47.66 & 59.26 & 6.28 & 1.0 & 16.0 & 28.00 & 62.0 & 396.0 \\
\hline
\end{tabular}

Notes: ICU: intensive care unit; ER: emergency room.

III, respectively, whereas the initial publication report a 6,16 , and $39 \%$ mortality for each group, respectively [16]. According to what was described in previous studies, there was a highly significant difference in mortality by separating patients with GAP score where the GAP III group exhibit a very high mortality rate in comparison with GAP I and III.

One of our major finding is that patients in GAP III present an annual rate of mortality of $42 \%$ and a median decline in DLCO of $30 \%$ of the initial absolute value enhancing the fact that these patients are at high risk. Moreover, the reduction in DLCO is higher in that group than in patient in GAP II and III . Therefore, we truly consider that this is our responsibility to accelerate the access to the anti-fibrotic therapies since pirfenidone has been shown to reduce the overall mortality in IPF patients.

In this context, biomarkers are highly needed in IPF as tools for differential diagnostic, predictor of the progression of the disease and treatment response [18]. Specifically in IPF, an early diagnostic is important to reduce as much as possible the disease progression. Ideally, biomarkers should easily be sampled (in opposition with BAL and surgical lung biopsy) and analyzed for a wide-spread utility in clinical practice.

\section{Disclosure statement}

No potential conflict of interest was reported by the authors.

\section{References}

[1] Raghu G, Collard HR, Egan JJ, et al. An official ATS/ ERS/JRS/ALAT statement: idiopathic pulmonary fibrosis: evidence-based guidelines for diagnosis and management. Am J Respir Crit Care Med. 2011;183(6):788-824.

[2] Guiot J, Corhay JL, Louis R. Idiopathic pulmonary fibrosis. Rev Med Liege. 2014;69(11):605-610.

[3] Raghu G, Weycker D, Edelsberg J, et al. Incidence and prevalence of idiopathic pulmonary fibrosis. Am J Respir Crit Care Med. 2006;174(7):810-816.

[4] Kaunisto J, Salomaa E-R, Hodgson U, et al. Idiopathic pulmonary fibrosis - a systematic review on methodology for the collection of epidemiological data. BMC Pulm Med. 2013;13(1):53.

[5] Travis WD, Costabel U, Hansell DM, et al. An Official American Thoracic Society/European Respiratory Society statement: update of the international multidisciplinary classification of the idiopathic interstitial pneumonias. Am J Respir Crit Care Med. 2013;188(6):733-748.

[6] Raghu G. Idiopathic pulmonary fibrosis: guidelines for diagnosis and clinical management have advanced from consensus-based in 2000 to evidence-based in 2011. Eur Respir J. 2011;37(4):743-746.

[7] Raghu G, Chen S-Y, Hou Q, et al. Incidence and prevalence of idiopathic pulmonary fibrosis in US adults 18-64 years old. Eur Respir J. 2016;48(1):179186.

[8] Gilani SR, Vuga LJ, Lindell KO, et al. CD28 downregulation on circulating CD4 T-cells is associated with poor prognoses of patients with idiopathic pulmonary fibrosis. PLoS ONE. 2010;5(1):e8959.

[9] King TE, Bradford WZ, Castro-Bernardini S, et al. A phase 3 trial of pirfenidone in patients with idiopathic pulmonary fibrosis. N Engl J Med. 2014;370(22):20832092.

[10] Richeldi L, du Bois RM, Raghu G, et al. Efficacy and safety of nintedanib in idiopathic pulmonary fibrosis. $\mathrm{N}$ Engl J Med. 2014;370(22):2071-2082.

[11] Maher TM. profileing idiopathic pulmonary fibrosis: rethinking biomarker discovery. Eur Respir Rev. 2013;22(128):148-152.

[12] Guiot J, Bondue B, Henket M, et al. Raised serum levels of IGFBP-1 and IGFBP-2 in idiopathic pulmonary fibrosis. BMC Pulm Med. 2016;16(1):86.

[13] Guiot J, Henket M, Corhay JL, et al. Sputum biomarkers in IPF: evidence for raised gene expression and protein level of IGFBP-2, IL-8 and MMP-7. In: Morty RE, editor. PLoS ONE. Vol. 12. San Francisco (CA): Public Library of Science; 2017. p. e0171344.

[14] Noble PW, Albera C, Bradford WZ, et al. Pirfenidone for idiopathic pulmonary fibrosis: analysis of pooled data from three multinational phase 3 trials. Eur Respir J. 2015;47(1):ERJ-00026.

[15] Raghu G, Rochwerg B, Zhang Y, et al. An official ATS/ ERS/JRS/ALAT clinical practice guideline: treatment of idiopathic pulmonary fibrosis. an update of the 2011 clinical practice guideline. Am J Respir Crit Care Med. 2015;192(2):e3-e19.

[16] LeyB, Ryerson CJ, Vittinghoff E, etal. A multidimensional index and staging system for idiopathic pulmonary fibrosis. Ann Intern Med. 2012;156(10):684-691.

[17] Kishaba T, Nagano H, Nei Y, et al. Clinical characteristics of idiopathic pulmonary fibrosis patients according to their smoking status. J Thorac Dis. 2016;8(6):11121120 .

[18] Guiot J, Moermans C, Henket M, et al. Blood biomarkers in idiopathic pulmonary fibrosis. Lung. 2017;195(3):273-280. 